يعتبر الاستثمار الزراعي أساس لنجاح التنمية الزراعية، حيث يعتبر الركيزة الأساسية لزيادة الإنتاج وكذلك الكاد

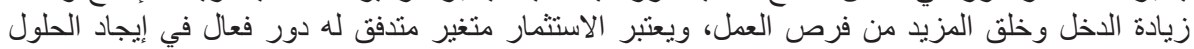

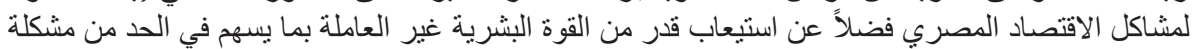

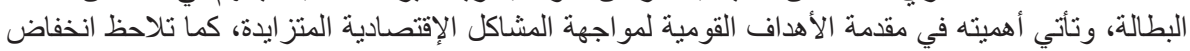

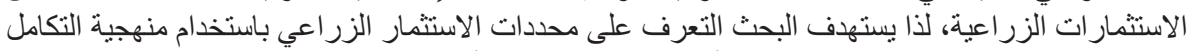

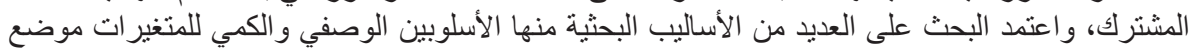

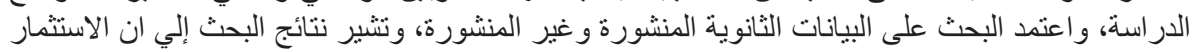

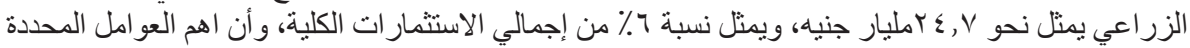

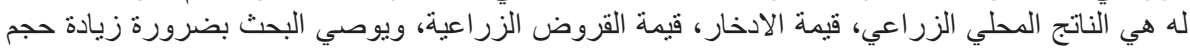

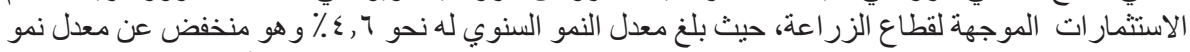

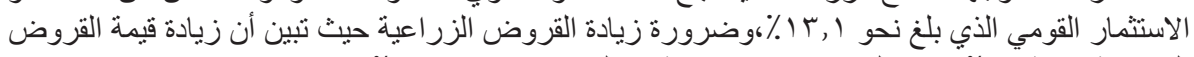

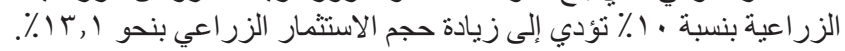

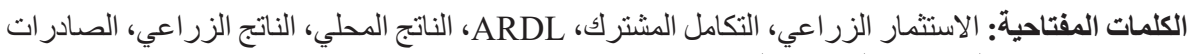

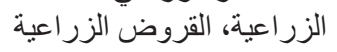

لللك تسعي الحكومة المصرية في الفترة الأخيرة إلي زيادة

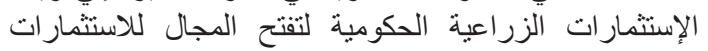

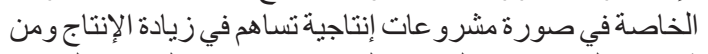

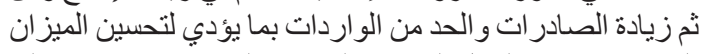

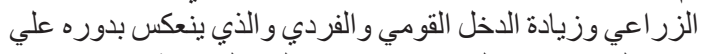

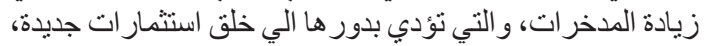

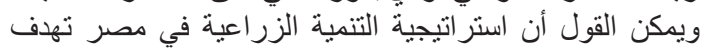

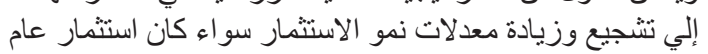

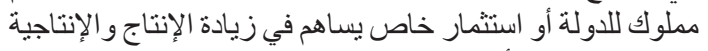

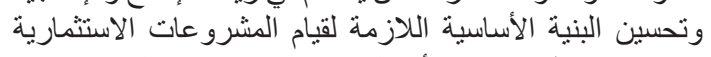

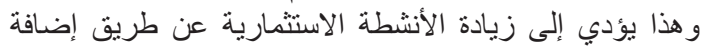
مشرو عات جديدة داخل البنيان الاقتصادي.

\section{المشكلة البحثية}

تكمن المشكلة البحثية في إنخفاض حجم الاستثمارات

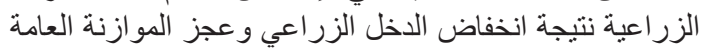

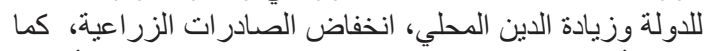

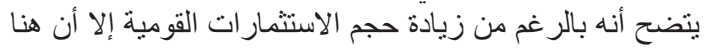

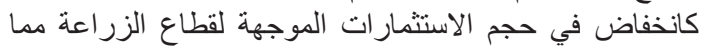

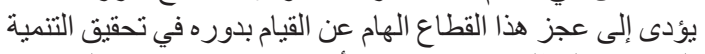

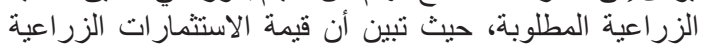

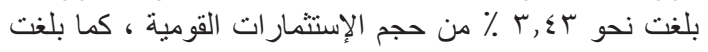

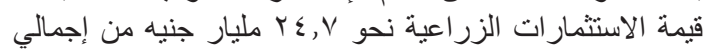

المقدمة

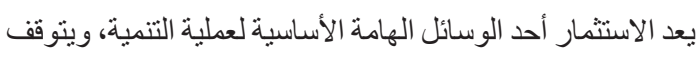

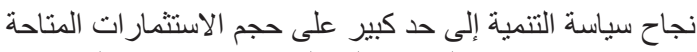

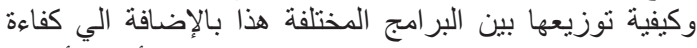

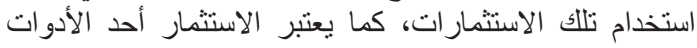

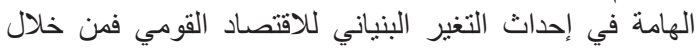

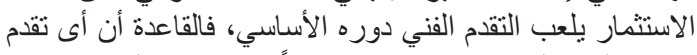

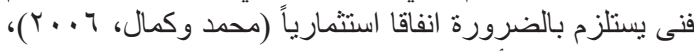

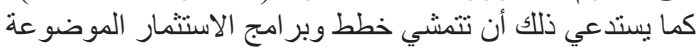

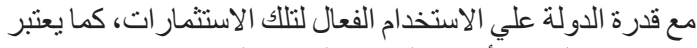

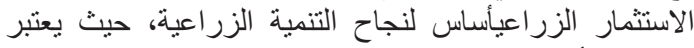

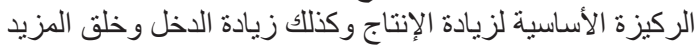

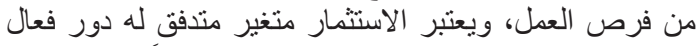

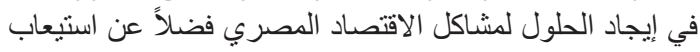

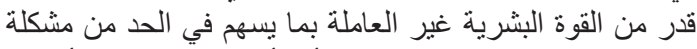

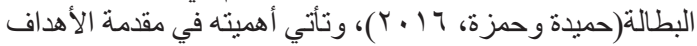

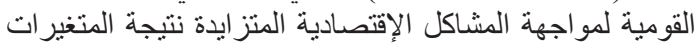

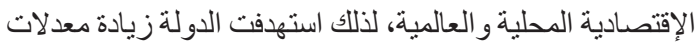

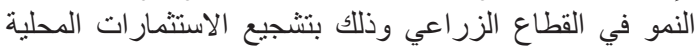

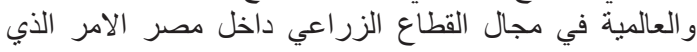

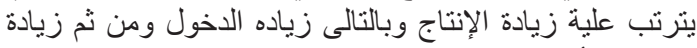

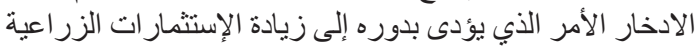

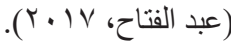

"Corresponding author: ahmedsalah4045@gmail.com DOI : $10.21608 /$ jsas.2020.18738.1182

Received:27/10/2019;Accepted:20/01/2020

C2020 National Information and Documentation Center (NIDOC) 


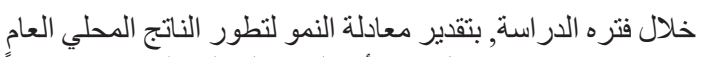

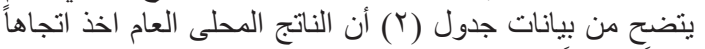

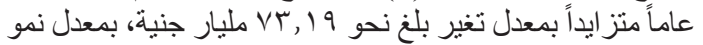

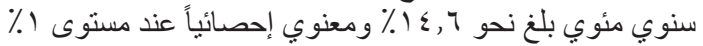

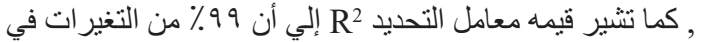
الناتج المحلى العام يرجع اثرها مدا إلي عنصر الزمن.

$$
\text { بـ تطور الناتج المحلي الخاص في مصر }
$$

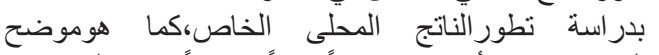

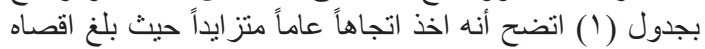

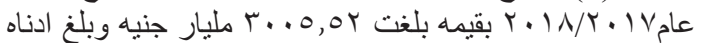

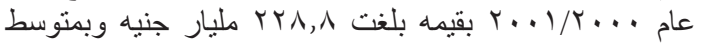

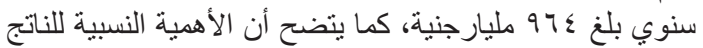

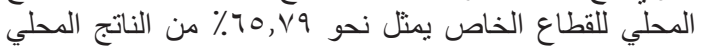

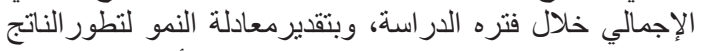

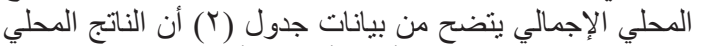

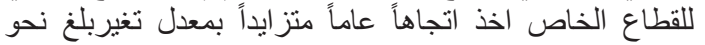

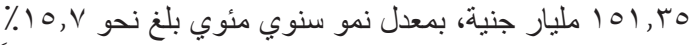

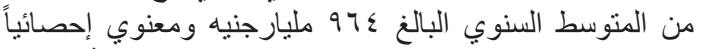

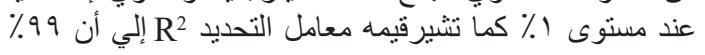

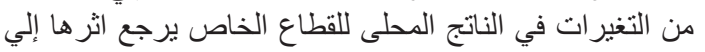

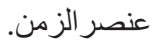
ثانياً:تطور الناتج المحلي الزراعي في مصر

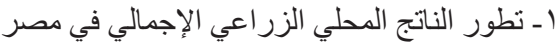

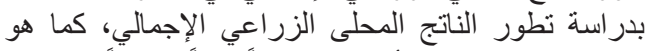

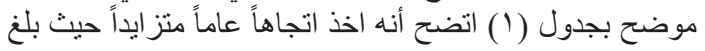

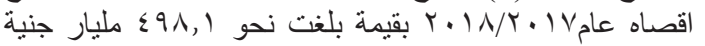

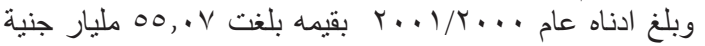

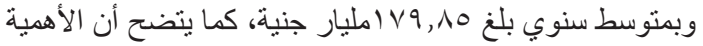

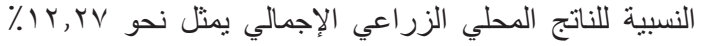

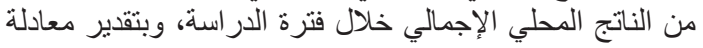

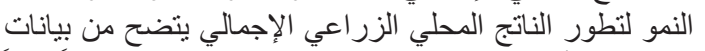

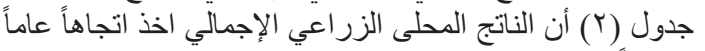

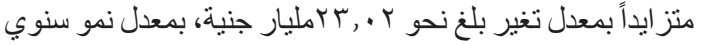

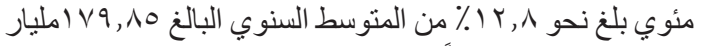

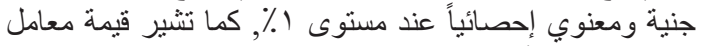

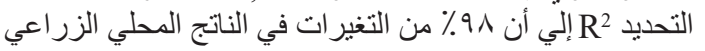
الإجمالي يرجع اثر ها إلي عنص عن النصر الزمن. r ـ تطور الناتج المحلي الزر اعي العام في مصر.

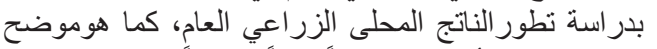

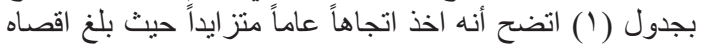

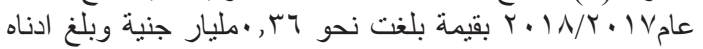

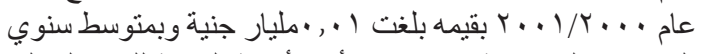

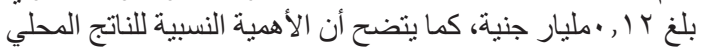

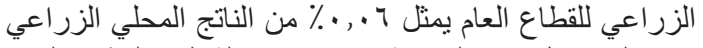

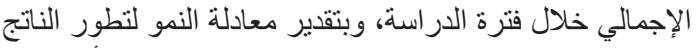

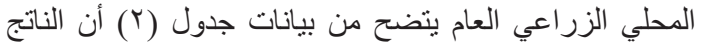

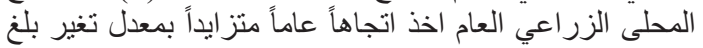

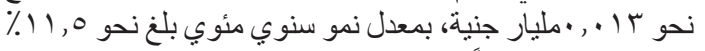

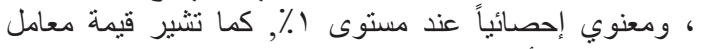

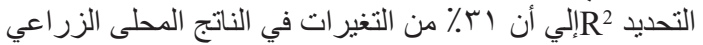
العام يرجع اثرها إلي عنصر الزيد من النغيرات

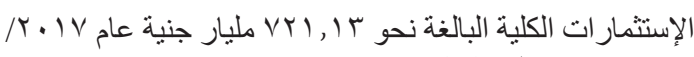

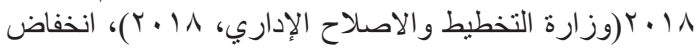

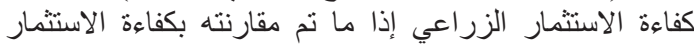

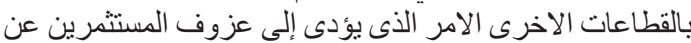
الاستثمار بقطاع الزرات الاعة.

الأهداف البحثبة

يهدف البحث بصفة رئيسية إلى دراسة محددات الاستثمار الزر اعي وذللك من خلال الأهداف الفر عية الآتية:

1- - در اسة تطور الاستثمار ات الكلية و الزر اعية العامة و الخاصة ونسبة مساهمته في الإستثمار ات الكلية خلال فترة الإلة الدراسة.

r- دراسة تطور الناتج المحلي الزراعي العام والخاص و الإجمالي ونسبة مساهمته في الناتج المحلي الإجمالي خلال العال

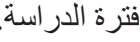

"- در اسة تطور كل من الصادرات و الواردات الزر اعية ونسبة

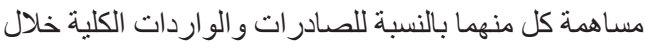
فترة الدر اسة.

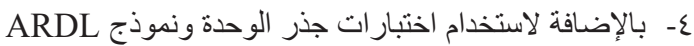
لاسة ألهم العو امل المحددة للاستثمار الزر اعي.

الأسلوب البحثى

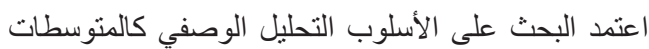

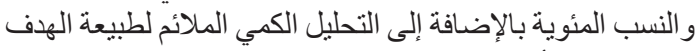

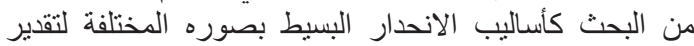

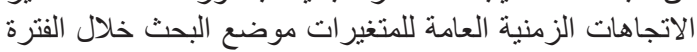

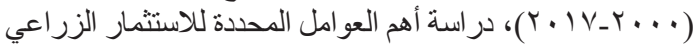

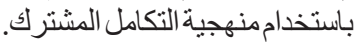
النتائج البحثية ومناقشتها أولاً: تطور الناتج المحلي المصري

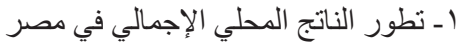

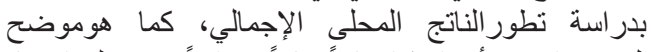

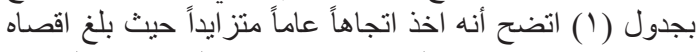

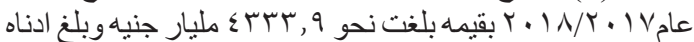

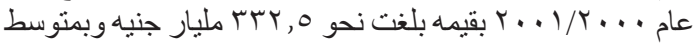

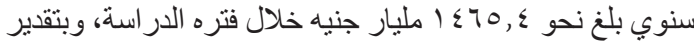

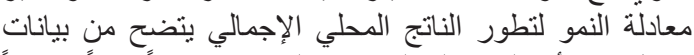

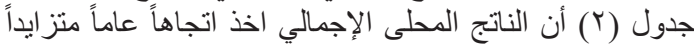

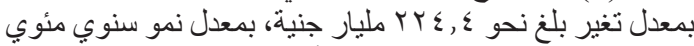

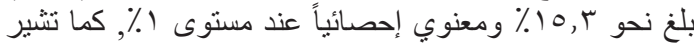

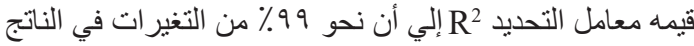
المحلى الإجمالي يرجع اثرها إلي عنصر الزئ الزمن.

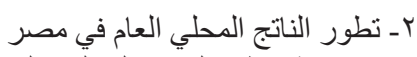

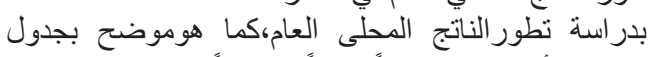

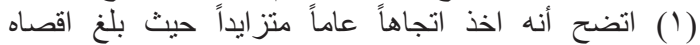

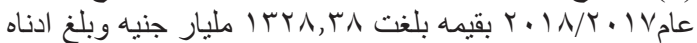

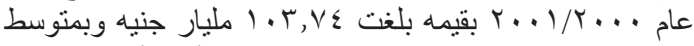

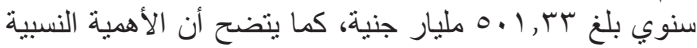

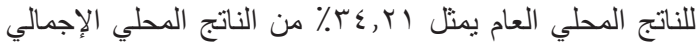




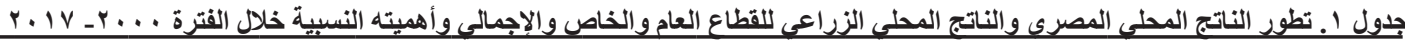

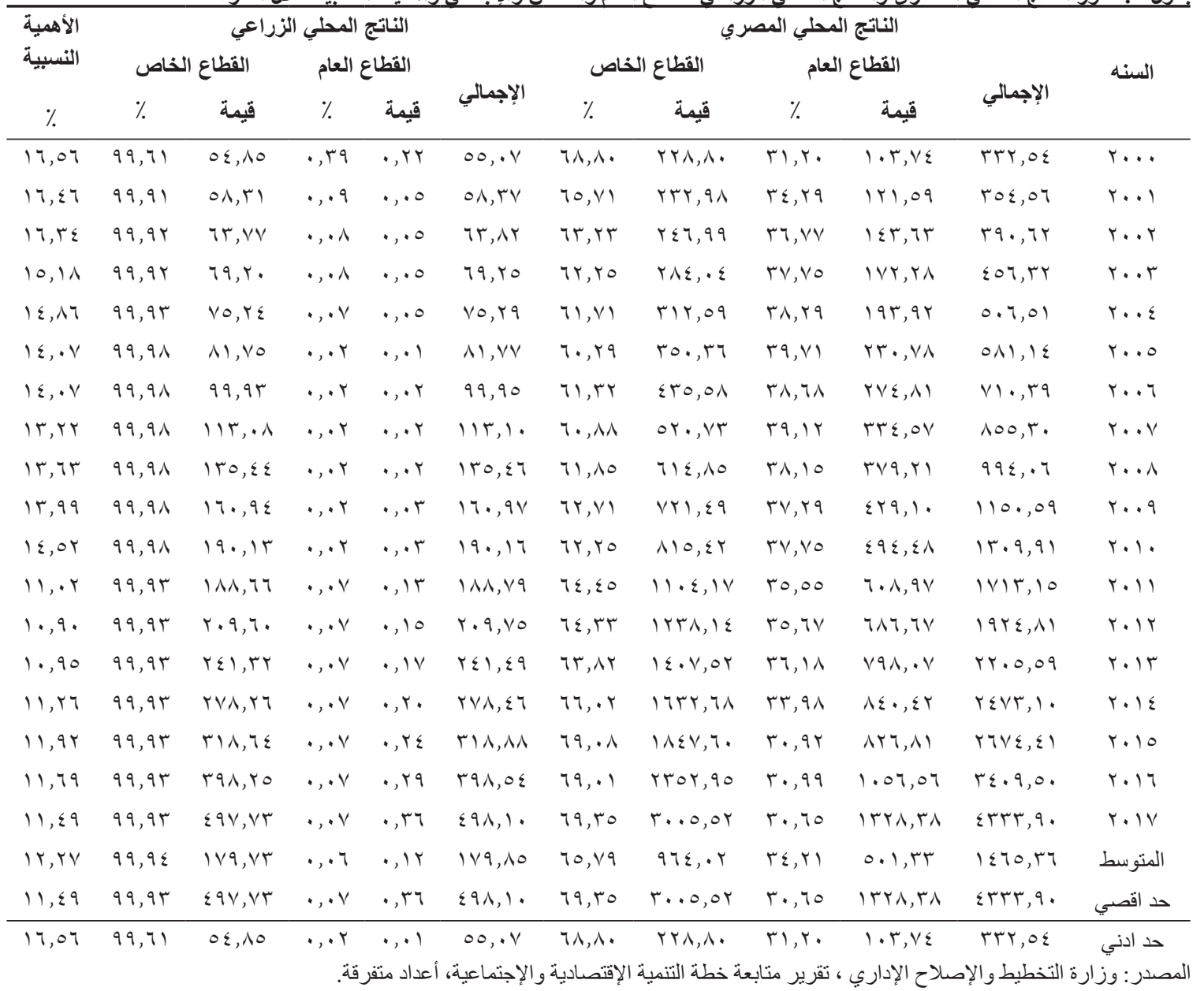

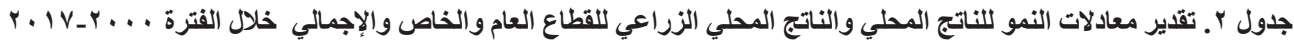

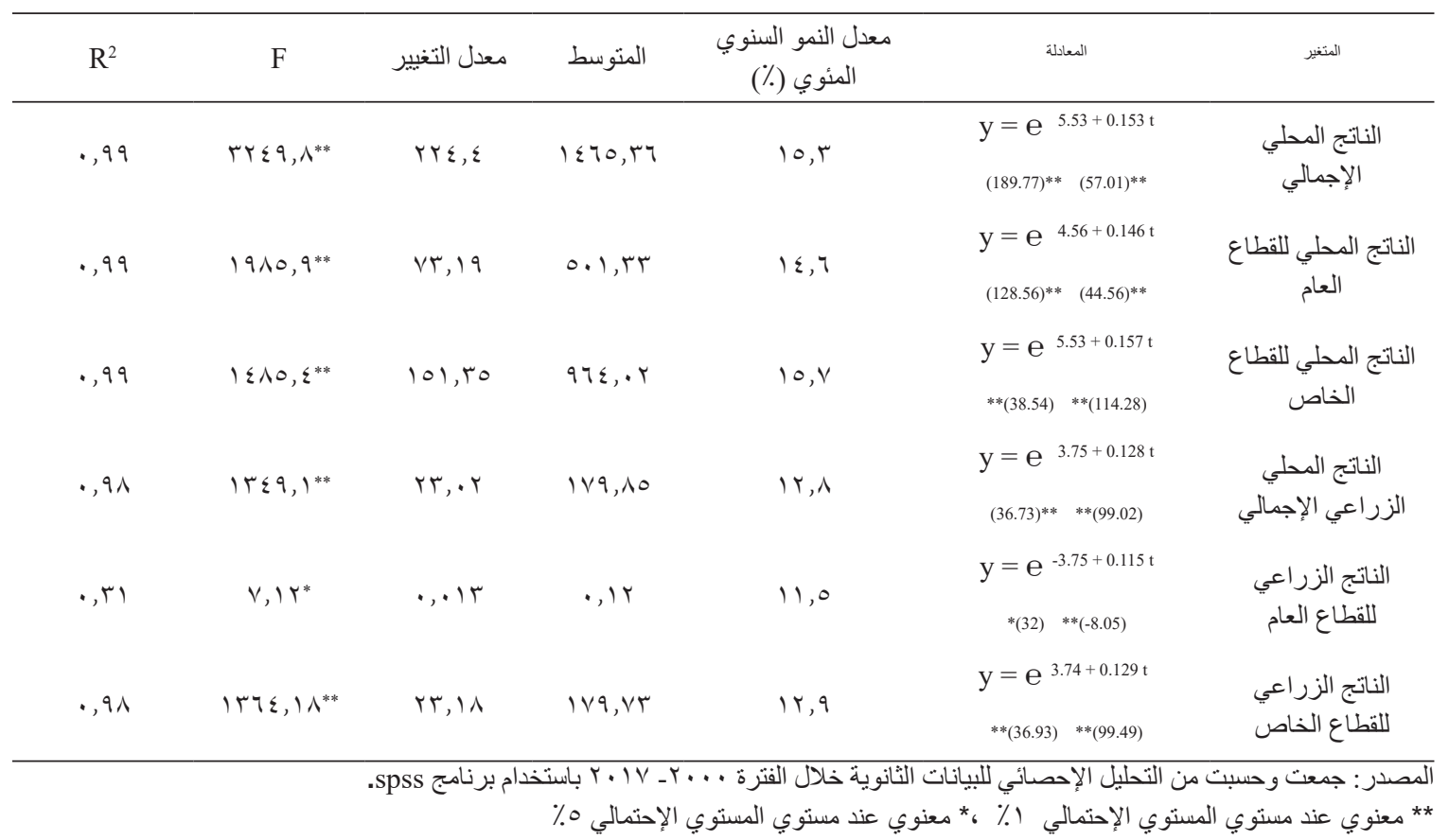

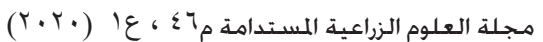


للقطاع الخاص اخذ اتجاهاً تصاعدياً حيث بلغت اقصاها عام

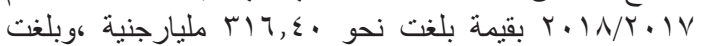

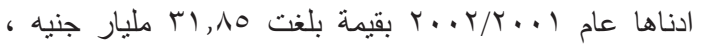

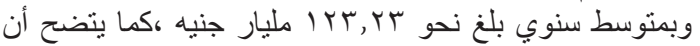

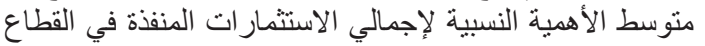

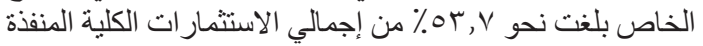

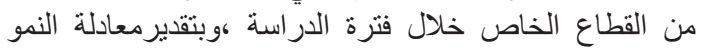

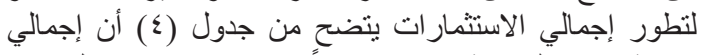

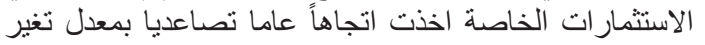

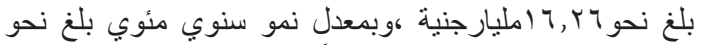

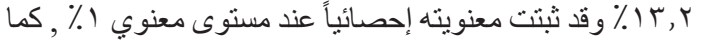

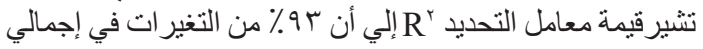

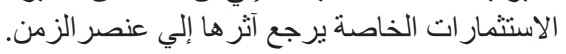

رابعاً: تطور الاستثمار ات الزر اعية خلال فترة الدر اسة إسة

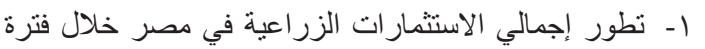
الدر اسة إنمال يتضح من شكل (r) أن إجمالي الاستثمار ات المنفذة في القطاع

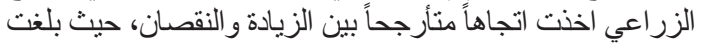

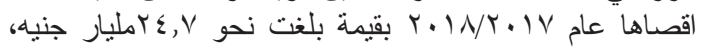

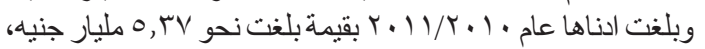

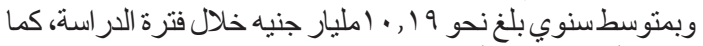

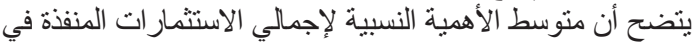

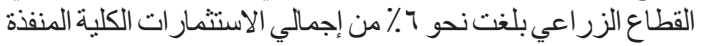

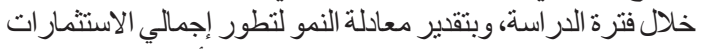

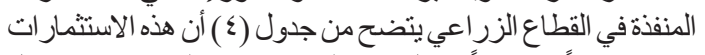

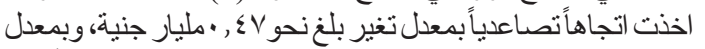

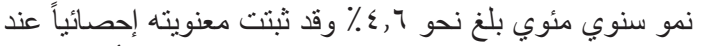

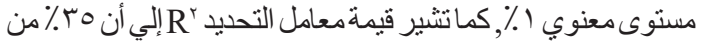

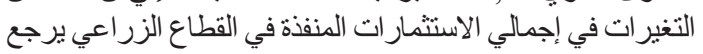

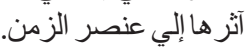

r- تطور إجمالي الاستثمارات الزراعية العامة في مصرخلال فترة الدر استة

يتضح من شكل (r) أن إجمالي الاستثمارات المنفذة في

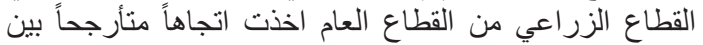

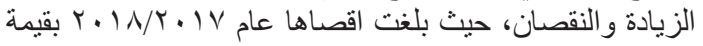

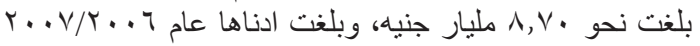

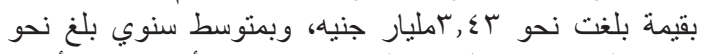
r r , V9

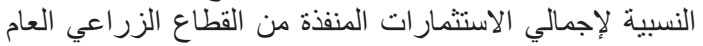

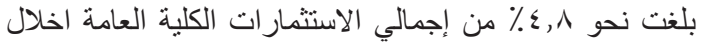

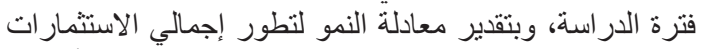

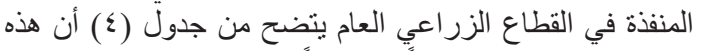

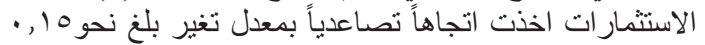

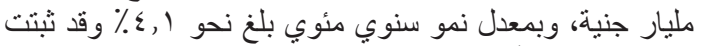

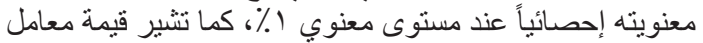

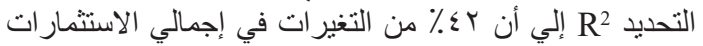
المنفذة في القطاع الزر اعي يرجع آثر ها إلي عنصر الزي الزمن. بـ تطور إجمالي الاستثمار ات الزر اعية الخاصة في مصر خلال

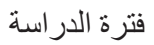

يتضح من شكل (Y) أن إجمالي الاستثمارات المنفذة في

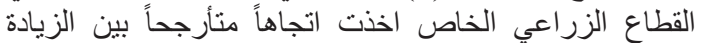

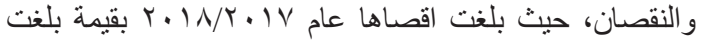

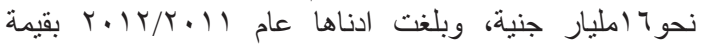

r-تطور الناتج المحلي الزر اعي الخاص في مصر .

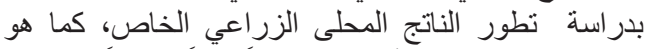

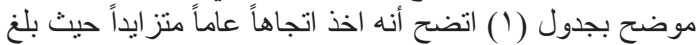

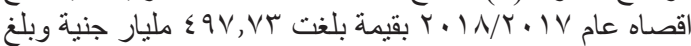

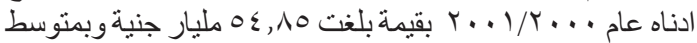

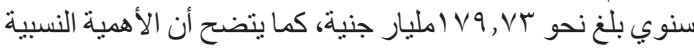

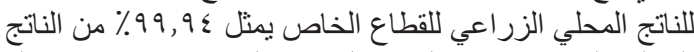

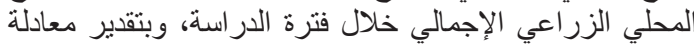

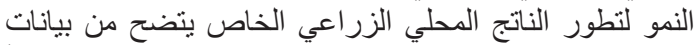

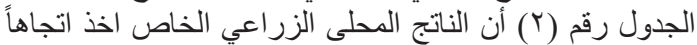

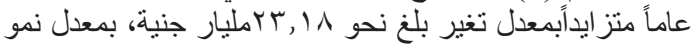

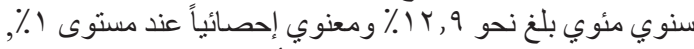

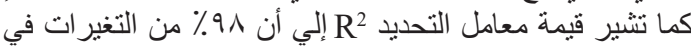

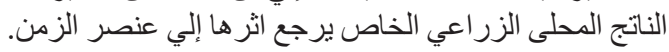

ثالثاً: تطور الاستثمار ات القومية خلال فترة الدر اسة بأنة

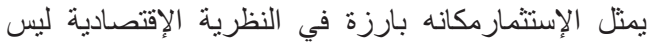

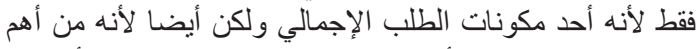

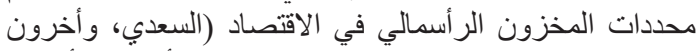

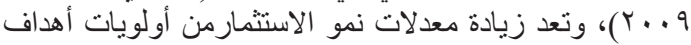

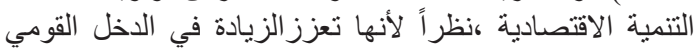

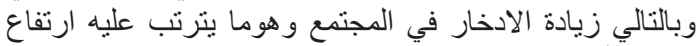

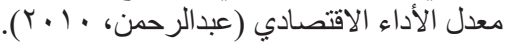

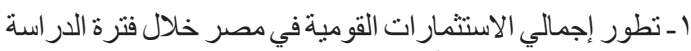

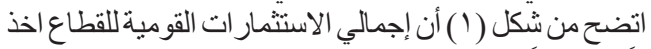

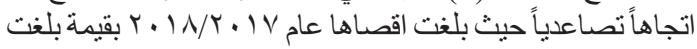

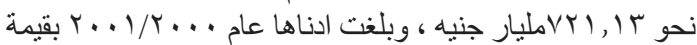

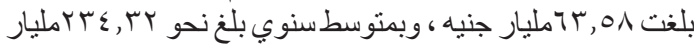

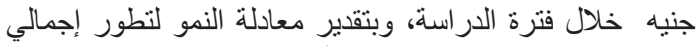

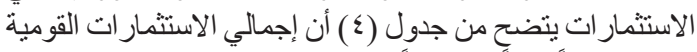

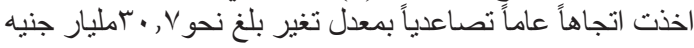

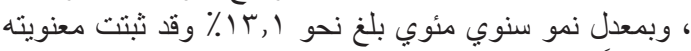

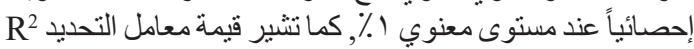

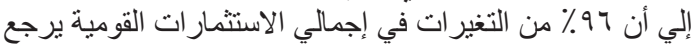

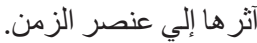

rـ تطور إجمالي الاستثمار ات القومية العامة في مصر خلال فترة الار اسة إنما

اتضح من شكل (1) أن إجمالي الاستثمار ات القومية للقطاع

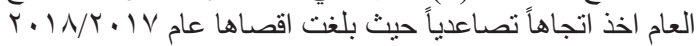

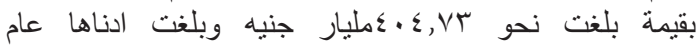

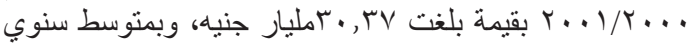

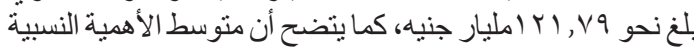

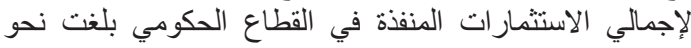

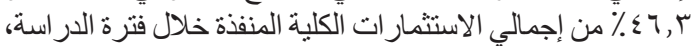

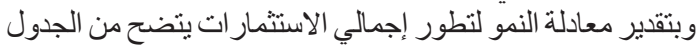

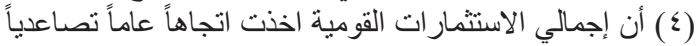

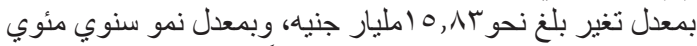

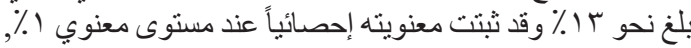

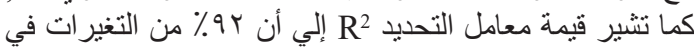

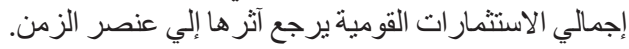
rـ تطور إجمالي الاستثمار ات القومية الخاصة في مصر خلال

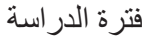

يتضح من شكل (1) أن إجمالي الاستثمارات القومية 


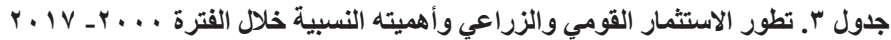

\begin{tabular}{|c|c|c|c|c|c|c|c|c|c|c|c|}
\hline \multicolumn{6}{|c|}{ الاستثمار الزراعي } & \multicolumn{5}{|c|}{ الاستثمار القومي } & \multirow{3}{*}{ السنه } \\
\hline \multicolumn{2}{|c|}{ الإجمالي } & \multicolumn{2}{|c|}{ 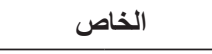 } & \multicolumn{2}{|c|}{ 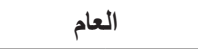 } & \multirow{2}{*}{ قالإجمالي } & \multicolumn{2}{|c|}{ 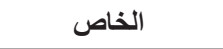 } & \multicolumn{2}{|c|}{ 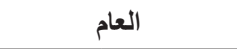 } & \\
\hline$\%$ & قيمة & $\%$ & قيمة & $\%$ & قيمة & & $\%$ & قيمة & $\%$ & قيمة & \\
\hline $1 r, 9$. & $\wedge, r$ & $\neg \varepsilon, V\urcorner$ & $0, M_{1}$ & ro,,$\leqslant \varepsilon$ & $r, \wedge q$ & & $0 ., 77$ & rY, Y & $\varepsilon q, r \varepsilon$ & r ו & $r \ldots$ \\
\hline $1 \wedge, Y^{\prime}$ & Ir, & $79,9 r$ & $\wedge, \uparrow$ & $r \cdot, \cdot \Lambda$ & $r, v$ & $7 V, 01$ & $\varepsilon v, \mid \wedge$ & M, 1, & or,$\wedge \varepsilon$ & ro, TV & $r \ldots 1$ \\
\hline$q, \varepsilon$. & $7, \varepsilon$ & $\varepsilon 9,79$ & r, & טו, & r, r & $7 \wedge, 1$ & $\{9, \leqslant 1$ & & $0 ., 7$. & $r \varepsilon, \varepsilon\rceil$ & $r \ldots r$ \\
\hline $9,0$. & $V, 07$ & or,, 91 & $\varepsilon$ & $\varepsilon v, \cdot q$ & $r, 0 r$ & $\vee 9,07$ & سד, & $r v, 1$ & or, & $\leqslant Y, \leqslant\rceil$ & $r$ \\
\hline$v, 79$ & $V, \varepsilon r$ & $O V, Y \wedge$ & $\varepsilon, Y_{0}$ & $\varepsilon r, V Y$ & $r, I V$ & $97, \leq 7$ & $\Sigma \Lambda, \mid r$ & $\leq 7, \leqslant r$ & $01, \wedge 1$ & $0,, \cdot \leq$ & $r \ldots \varepsilon$ \\
\hline 7,90 & $\wedge, \cdot \varepsilon$ & $70,1 \mathrm{~V}$ & $0, r \leq$ & r & $r, \Lambda$ & $110, v \leq$ & oV,r & אז,דר & $\varepsilon r, V$ & $\leqslant q, \leqslant r$ & r. . o \\
\hline $0, \cdot 1$ & $v, \vee q$ & $\backslash \wedge, \wedge 1$ & 0,14 & 41,19 & $r, \varepsilon r$ & $100, \pi \leq$ & $7 r, T \leq$ & qV, r & rV, & $0 \wedge, \cdot \leq$ & $r \ldots r$ \\
\hline$\varepsilon, \cdot \varepsilon$ & $\Lambda, \cdot V$ & $7 \varepsilon, 7 \wedge$ & $0, Y Y$ & r r & $r, \wedge 0$ & $199,0 \mathrm{r}$ & $7 \varepsilon, 79$ & $1 r q, \cdot 1$ & ו & $V_{\cdot}, \leq 0$ & $r . r v$ \\
\hline$\Gamma, \Sigma \wedge$ & $7, \wedge\urcorner$ & $7 \cdot, \cdot 7$ & $\varepsilon, 1 Y$ & $r q, q \leq$ & $r, V \leq$ & $19 v, 1 \leq$ & $\varepsilon \Lambda, \varepsilon \Gamma$ & $90, \leqslant \wedge$ & $01,0 \mathrm{~V}$ & 1.1, & $r \cdot \wedge$ \\
\hline$r, 91$ & $7, \vee \vee \varepsilon$ & $O V, \Sigma Y$ & $r, \wedge v$ & $\varepsilon r, V T$ & $r, \wedge \wedge$ & سג,וזr & $0 \varepsilon, 7 V$ & $M Y T, V \leq$ & س & $1.0, .9$ & $r .9$ \\
\hline$r, 9 \wedge$ & $7, \wedge \Gamma$ & Or, Ir & $r, 0 T$ & $\varepsilon \Lambda, \cdot r$ & $r, r \wedge$ & rrq, $\cdot V$ & 71,10 & $1 \leq 1,7 \wedge$ & rᄉ, 10 & NV,rq & $r \cdot 1$. \\
\hline$r, 1 \wedge$ & $0, T V$ & $0 ., Y \wedge$ & $r, V$ & $\varepsilon q, V r$ & $Y, T V$ & $r \leq \tau, \cdot V$ & qu, & 10r,or & (v, T) & $9 r, 00$ & $r .11$ \\
\hline$r, \varepsilon V$ & $\wedge, \Gamma \wedge$ & $\neg \varepsilon, \wedge$. & $0, \Sigma T$ & ro, r. & $r, 90$ & $r \leq 1,7)$ & $7 \cdot$ I I & $|\leq 0, V|$ & rq, 79 & 90,9 & $r \cdot 1 r$ \\
\hline$\varepsilon, \Gamma q$ & חד, 11 & T & $v, \varepsilon \wedge$ & ro, T/ & $\varepsilon, 10$ & r.o, 9 & سז, & $10 \varepsilon, 7 r$ & $\varepsilon 1,7 V$ & $11 \cdot, \leqslant V$ & r. \\
\hline$\varepsilon, \cdot r$ & $|r, \varepsilon|$ & 71,10 & $\wedge, r$ & rᄉ, ^o & $0, Y 1$ & Ir,VIr & $00, V_{1}$ & 110,94 & $\varepsilon \varepsilon, r q$ & $1 \leqslant v, v q$ & $r .1 \leq$ \\
\hline$\varepsilon, 10$ & $17, \mathrm{r} \wedge$ & $79, \cdot \varepsilon$ & $11, r \leq$ & $r \cdot, 97$ & $0, \cdot \varepsilon$ & rqr, $\varepsilon$ & Or, VY & $r 1 \cdot, 7 r$ & $\varepsilon \neg, Y \wedge$ & $|\wedge|, \leqslant Y$ & $r .10$ \\
\hline$r, r v$ & $\mid V, r \varepsilon$ & $70,1 V$ & $11, r$ & $r \varepsilon, \lambda r$ & $7, \cdot \leq$ & $0 \mid \leq, T_{1}$ & $\leqslant 1,0$. & YT, & $0 \wedge, 0$. & r., , & $r .17$ \\
\hline$r, \varepsilon r$ & $r \varepsilon, V$ & $T \varepsilon, V \wedge$ & 17 & ro, r & $\wedge, \vee$ & VYI, Ir & $\varepsilon r, \wedge \wedge$ & צו, & OT, Ir & $\varepsilon \cdot \varepsilon, V T$ & $r .1 \mathrm{~V}$ \\
\hline $0, \cdot \varepsilon$ & $1 \cdot, 11$ & $7 \cdot, 9$. & $7, r q$ & 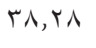 & $r, v q$ & rTE,Tr & • r, & سז, & $\varepsilon 0, V \leq$ & $111,1$. & المتوسط \\
\hline$\Gamma, \Sigma T$ & $r \varepsilon, V$ & \urcorner$\varepsilon, \vee \wedge$ & 17 & ro, rr & $\wedge, \vee$ & VYI, Ir & $\varepsilon r, \wedge \wedge$ & TI & $07,1 Y$ & $\varepsilon \cdot \varepsilon, V T$ & حد اقصي \\
\hline$\wedge, \leqslant 0$ & $0, T V$ & $0 ., Y \wedge$ & $r, V$ & $\varepsilon 0, Y_{0}$ & $r, \varepsilon r$ & & $0 ., .9$ & M, 1, & $\varepsilon q, r \varepsilon$ & r, rv & حد ادني \\
\hline
\end{tabular}

المصدر: وزارة التخطيطو الإصلاح الإداري، تقرير متابعة خطة التتميةالإقتصادية و الإجتماعية، أعداد متفرقة.

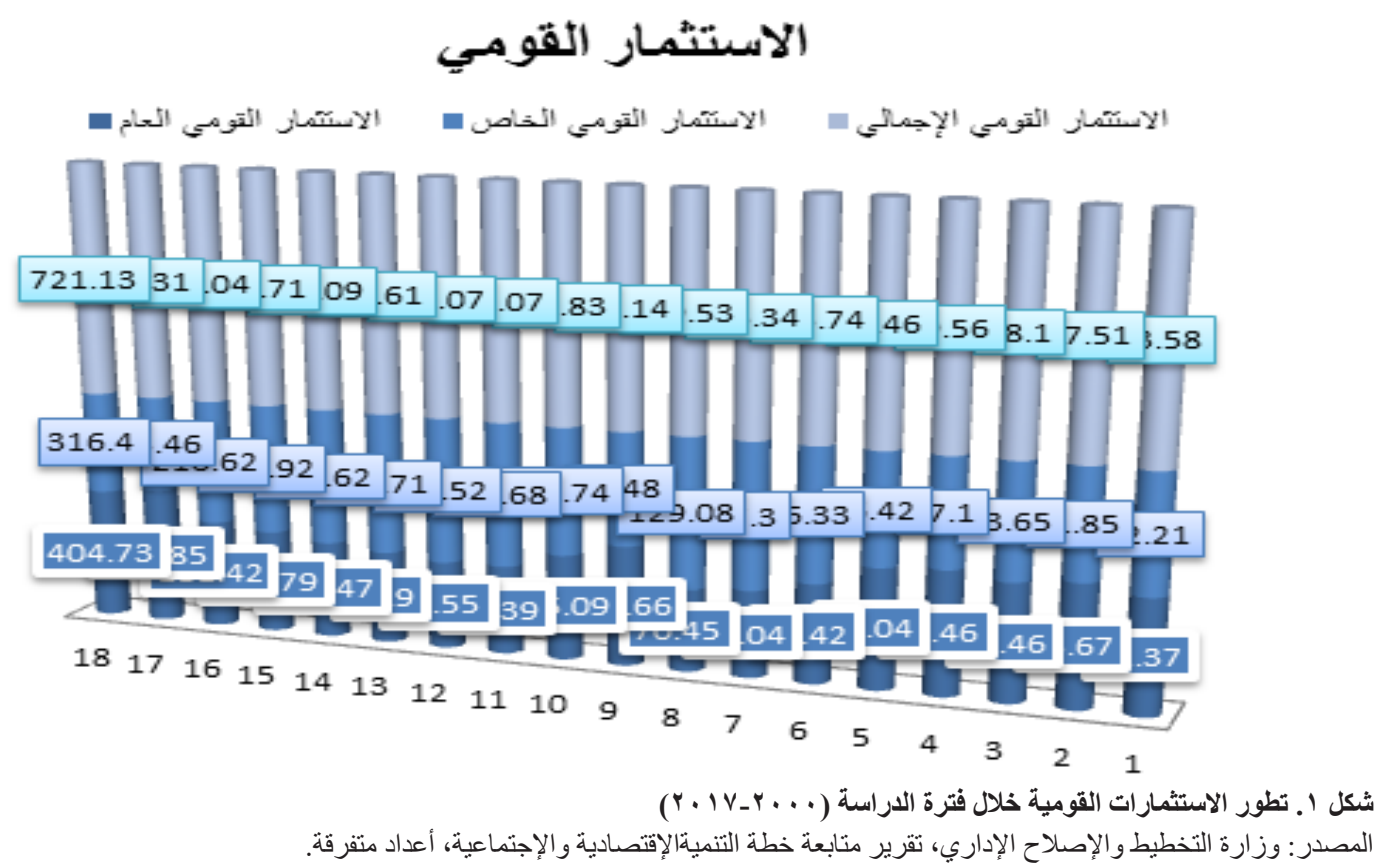

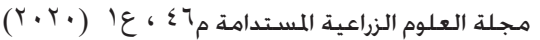




\section{الاستثمار الزراعي}

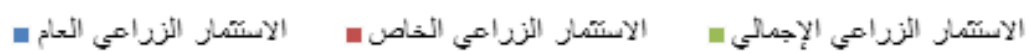

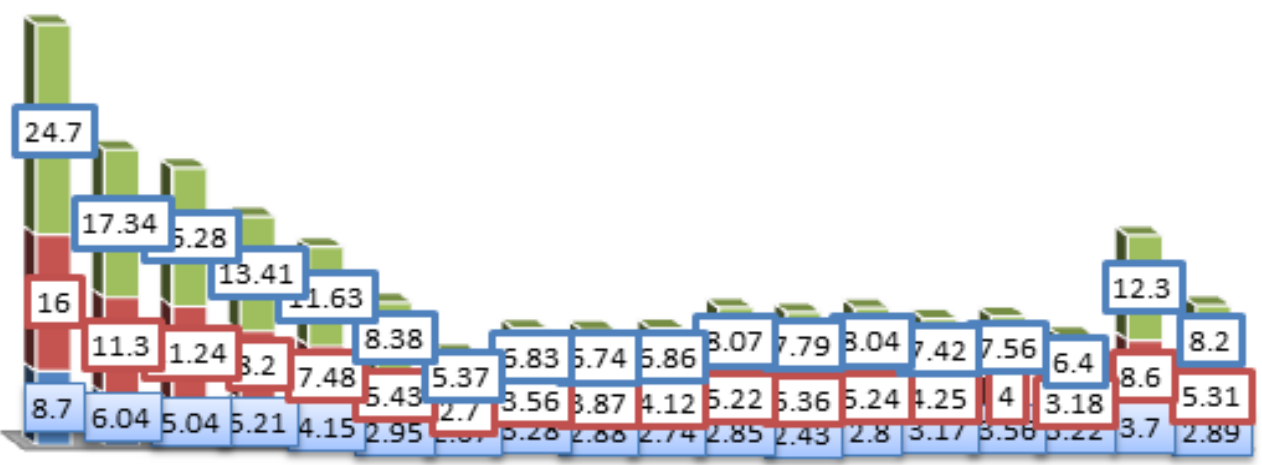

$\begin{array}{llllllllllllllllll}18 & 17 & 16 & 15 & 14 & 13 & 12 & 11 & 10 & 9 & 8 & 7 & 6 & 5 & 4 & 3 & 2 & 1\end{array}$

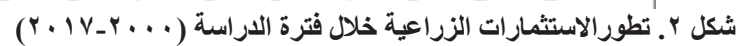

المصدر : وزارة التخطيط و الإصلاح الإداري، تقرير متابعة خطة التنمية الإقتصادية و الإجتماعية، أعداد متفرقة

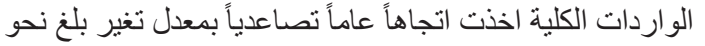

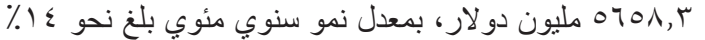

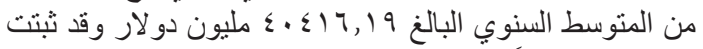

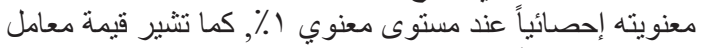

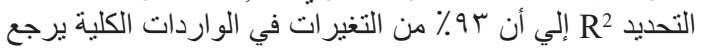

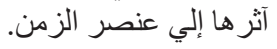

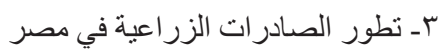

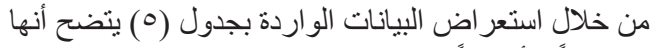

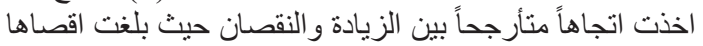

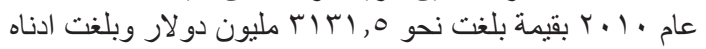

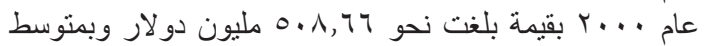

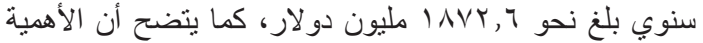

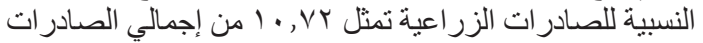

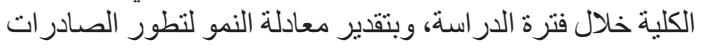

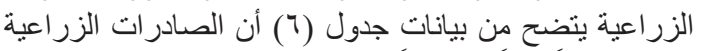

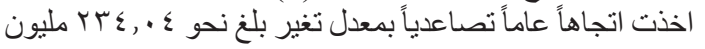

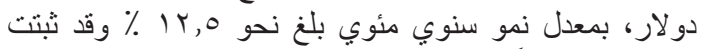

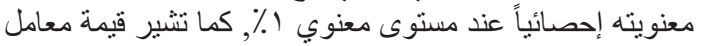

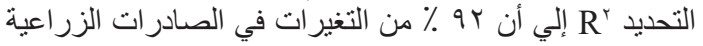
يرجع آثرها إلي عنصر الزي الزمن.

ع ـتطور الواردات الزر اعية في مصر.

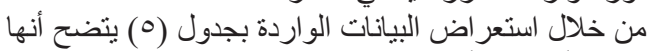

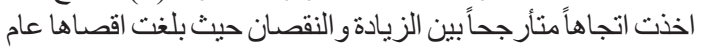

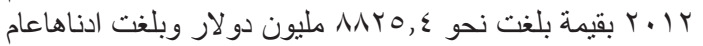

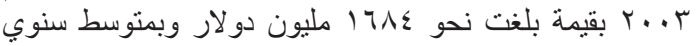

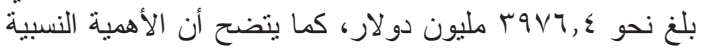

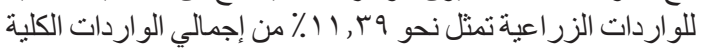

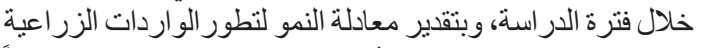

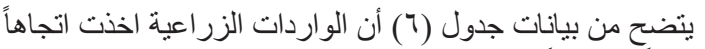

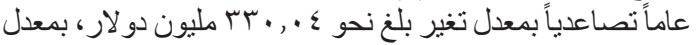

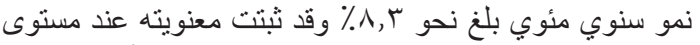

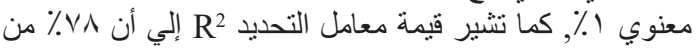
التغيرات في الواردات الزر اعية يرجع آثرها إلي عنصر الزئ الزمن.

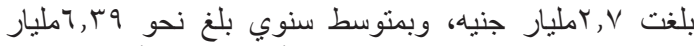

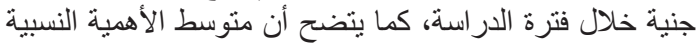

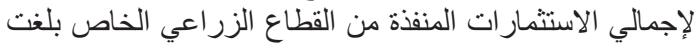

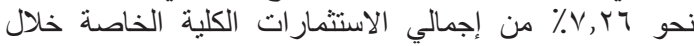

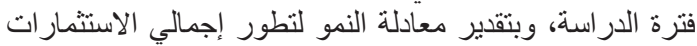

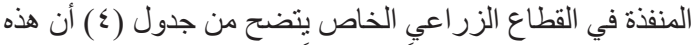

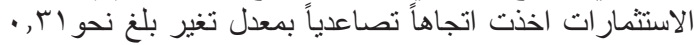

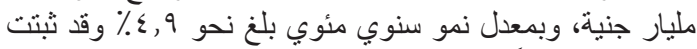

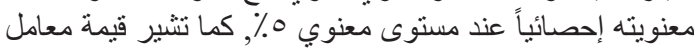

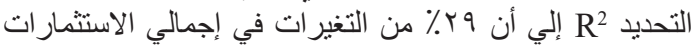

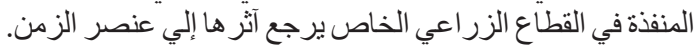

خامساً :تطور الصادرات و الواردات الكلية و الزر اعية في مصر

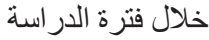

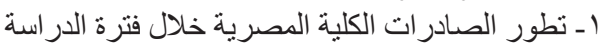

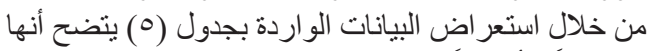

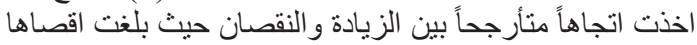

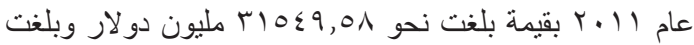

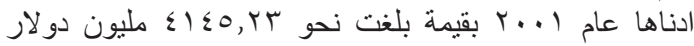

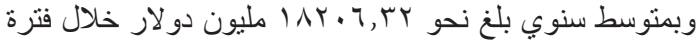

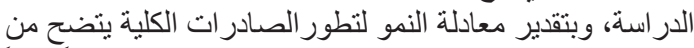

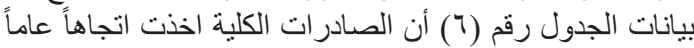

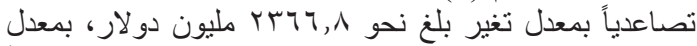

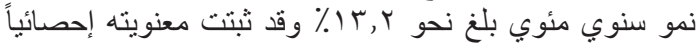

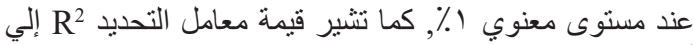
أن 19 ٪ من التغيرات في الصادرات الكلية يرجع آثرها إلي التي

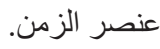

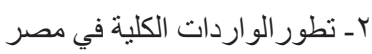

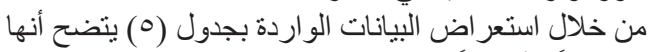

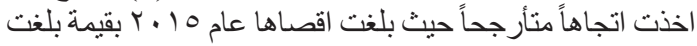

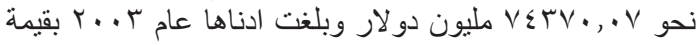

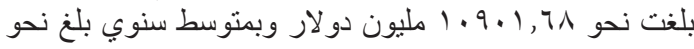

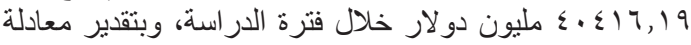

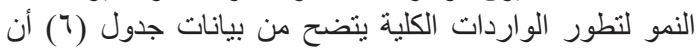

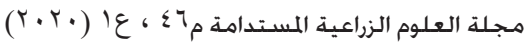


جدول ؛ ـ تقدير معادلة النمو للاستثمار القومي والزراعي للقطاع العام والخاص والإجمالي خلال فترة الدراسة

\begin{tabular}{|c|c|c|c|c|c|c|}
\hline $\mathbf{R}^{2}$ & $\mathbf{F}$ & معدل التغيير & المتوسط & $\begin{array}{c}\text { السنوي المعدوي النموي } \\
\text { (\%) }\end{array}$ & المعادلة & المتغير \\
\hline$\cdot, 97$ & $r ч, \vee 0^{* *}$ & $r \cdot, v$ & $r r \varepsilon, r Y$ & $\mid r, 1$ & $\begin{array}{c}y=e^{3.97+0.131 t} \\
(53.5)^{* *} \quad(19.17)^{* *}\end{array}$ & الاستثمار القومي \\
\hline$\cdot, 94$ & $\mid V \varepsilon,+r^{* *}$ & $10, \lambda r$ & $|r|, v q$ & IT & $\begin{array}{c}y=e^{3.21+0.13 t} \\
(30.17)^{* *}(13.19)^{* *}\end{array}$ & الاستثمار العام \\
\hline • , 94 & $Y .9,01^{* *}$ & $17, Y^{7}$ & IrT, & $M, r$ & $\begin{array}{c}y=e^{3.34+0.132 t} \\
(33.73)^{* *}(14.47)^{* *}\end{array}$ & الاستثمار الخاص \\
\hline • tro & $\wedge, \wedge 0^{* *}$ & $\cdot, \leqslant V$ & $1 \cdot, 19$ & $\varepsilon, 7$ & $\begin{array}{c}y=e \quad 1.8+0.046 t \\
(10.81)^{* *} \quad(2.98)^{* *}\end{array}$ & الاستثمار الزر اعي \\
\hline$\cdot, \varepsilon r$ & $11, \wedge r^{* * *}$ &., 10 & $r, v q$ & $\varepsilon, 1$ & $\begin{array}{c}\mathrm{y}=\mathrm{e}^{0.88+0.041 \mathrm{t}} \\
(6.81)^{* *}(3.43)^{* *}\end{array}$ & الاستثمار الزراعي العام \\
\hline$\cdot, r q$ & $7, V^{*}$ & . & $7, r 9$ & $\varepsilon, 9$ & $\begin{array}{c}\mathrm{y}=\mathrm{e}^{1.26+0.049 \mathrm{t}} \\
(6.16)^{* *}(2.59)^{*}\end{array}$ & الاستثمار الزراعي \\
\hline
\end{tabular}

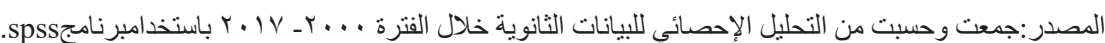

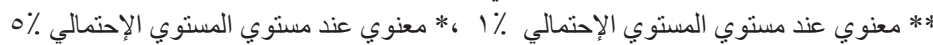

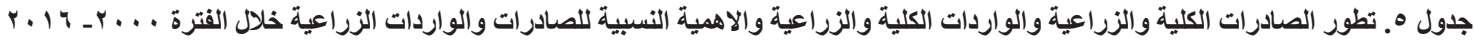

\begin{tabular}{|c|c|c|c|c|c|c|}
\hline \multicolumn{2}{|c|}{ واردات زراعية } & \multicolumn{2}{|c|}{ صادرات زراعية } & \multirow{2}{*}{ واردات كلية } & \multirow{2}{*}{ صادرات كلية } & \multirow{2}{*}{ السنـه } \\
\hline$\%$ & قيمة & $\%$ & قيمة & & & \\
\hline $1 \leq, \cdot 1$ & $190 \wedge, \pi 1$ & $1 \cdot, 1$ & $0.1,77$ & 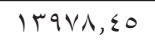 & $\leqslant v 11, \leqslant 9$ & $r \ldots$ \\
\hline 10,1 & $|9 r|, \lambda \varepsilon$ & $1 r, v q$ & or., 1 r. & IrVYA,rq & $\Sigma 1 \leqslant 0, r r$ & $r \ldots r$ \\
\hline$I V, Y_{0}$ & r100,1T & $1 \varepsilon, r \varepsilon$ & $7 \times 1, .0$ & $1 r \leqslant 97, \cdot r$ & $\sum T \vee A, 1$ & $r \ldots r$ \\
\hline $10, \leqslant 0$ & $17 \wedge, 97$ & $1,0,09$ & $V \vee Y, r$ & $1 \cdot 9 \cdot 1,71$ & $7171, \cdot 1$ & $r \ldots r$ \\
\hline $1 r, r^{\prime}$ & $1 v \cdot 0, \leqslant 0$ & $1 \leq, r q$ & $|1 \cdot \varepsilon, 7|$ & $|r \wedge r u, v|$ & VTVV,TY & $r \ldots \varepsilon$ \\
\hline $1 r, 17$ & $r \varepsilon \cdot V, q \leq$ & $\Lambda, T r$ & $q 1 V, q r$ & $191 \cdot v, 9 \leq$ & $1 . \tau \leqslant r, r_{0}$ & $r \ldots o$ \\
\hline $11, \cdot 1$ & หrาq, $\varepsilon \varepsilon$ & T, T & 100,17 & $r \cdot 7 \cdot 0,91$ & $|r V| 0, \varepsilon \wedge$ & $r \ldots T$ \\
\hline $\mid r, \leq \Lambda$ & $T \neg \leq 7, T \leq$ & $V, \varepsilon r$ & $M \cdot r, \leq 0$ & $Y V .0 \leqslant, Y T$ & $1711 \cdot, 1 \leq$ & $r \ldots v$ \\
\hline 9,11 & $\leqslant \wedge 19, \varepsilon r$ & $V, 97$ & $r \cdot \wedge q, v \leq$ & orvqr, rq & rtros, IV & $r \ldots \wedge$ \\
\hline $9, \times 7$ & $\varepsilon r \wedge q, q r$ & Ir,YY & YqTV,T) & $\varepsilon \leqslant 90 \wedge, r V$ & $r \leqslant r \cdot T, T_{0}$ & $r \ldots q$ \\
\hline $1 \cdot, 71$ & & $11, \leq 0$ & 0, & Or.TV,ru & YVTON,TT & r.1. \\
\hline$M, r$ & NTI,,$\Delta T$ & 9,09 & $r \cdot r_{0}, T V$ & $T r K \mid 1,09$ & $r_{10} 0 \leqslant 9,01$ & $r .11$ \\
\hline$|r, \varepsilon|$ & $\Lambda \wedge Y_{0}, \varepsilon Y$ & $9,1 \vee$ & צד,ז'ז & $v 1 \cdot 9 \Lambda, \cdot r$ & YЯYтะ,Y & $r \cdot I r$ \\
\hline$V, V \varepsilon$ & $0 I M T, V A$ & $9,9 \wedge$ & rATV,. & ITYVA, VA & YAVTA, rV & $r \cdot i r$ \\
\hline $0,7 \varepsilon$ & E)Tr,9V & $1 \cdot, V Y$ & r90, 10 & VTAYA, VV & $r v 0 \leq r, \cdot r$ & r.lE \\
\hline 0,01 & $\leq 1 \ldots, \cdot 1$ & $\mid r, q \wedge$ & rAOY, Y $\varepsilon$ & $v \leqslant r v \cdot, \cdot v$ & $r \mid 9 V_{\cdot}, \leq 7$ & $r .10$ \\
\hline$v, \wedge \wedge$ & $\varepsilon 0 \vee 0, \wedge r$ & $1 ., 9 r$ & rד97, & ON.OY,T & $r \leq 79 \wedge, \wedge \wedge$ & $r .17$ \\
\hline $11, r q$ & 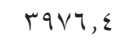 & $1 \cdot, V r$ & $1 \wedge V Y, T$ & $\varepsilon \cdot\{17,19$ & $1 A r \cdot T, r r$ & المتوسط \\
\hline$I V, Y_{0}$ & ANYO, $\varepsilon$ & $1 \varepsilon, r q$ & סוזוץ & $v \leqslant r v \cdot, \cdot v$ & $\Gamma_{10} 0 \leqslant 9,01$ & حد أقصي \\
\hline 0,01 & $171 \varepsilon$ & T, ד & $0.1,77$ & $1 \cdot 9 \cdot 1,71$ & $\varepsilon 1 \leqslant 0, r r$ & حد أدني \\
\hline
\end{tabular}

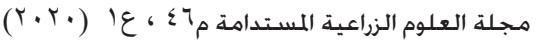




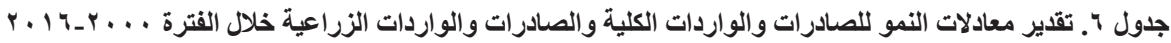

\begin{tabular}{|c|c|c|c|c|c|c|}
\hline $\mathrm{R}^{2}$ & $\mathrm{~F}$ & معدل التغيير & المتوسط & معدل النمو السنوي & المعادلة & المتغير \\
\hline$\cdot, 19$ & $7 ., 01 * *$ & אזדז, & $1 \wedge r \cdot T, r r$ & Ir, & $\begin{array}{l}y=8.4+0.132 t \\
(48.6)^{* *}(7.78)^{* *}\end{array}$ & الصـادر ات الكلية \\
\hline • & $1 \cdot 1, \cdot r^{* * *}$ & $0701, r$ & $\varepsilon \cdot\{17,19$ & $1 \varepsilon$ & $\begin{array}{l}\mathrm{y}=\mathrm{e}^{9.14+0.14 \mathrm{t}} \\
(65.18)^{* *} \quad(10.05)^{* *}\end{array}$ & الو اردات الكلية \\
\hline$\cdot, 9 T$ & $\wedge 1,19 * *$ & $r T \leq, \cdot A$ & IAVY,T & $1 r, 0$ & $\begin{array}{c}\mathrm{y}=\mathrm{e}^{6.21+0.125 \mathrm{t}} \\
(43.53)^{* *} \quad(9.01)^{* *}\end{array}$ & الصادر ات الزر اعية \\
\hline$\cdot, \mathrm{VA}$ & $r \leqslant, \Gamma^{* * *}$ & צ.., •."ז & 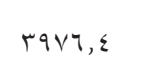 & $\Lambda, r$ & $\begin{array}{l}y=e \quad 7.41+0.083 t \\
(42.92)^{* *}(4.93)^{* * *}\end{array}$ & الواردات الزر اعية \\
\hline
\end{tabular}

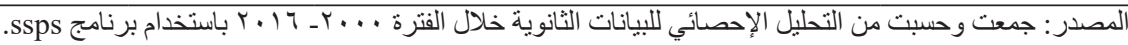

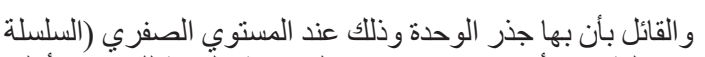

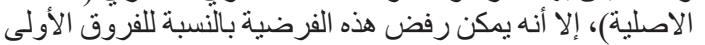

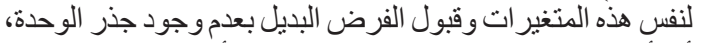

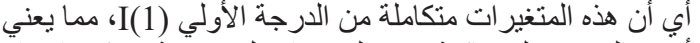

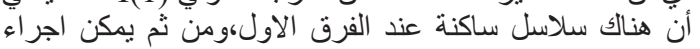

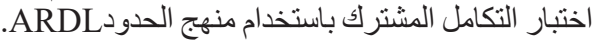

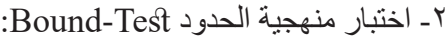
يتم هذا الاختبار بعد فحص الحدار استقرار السلاسل الزمنية

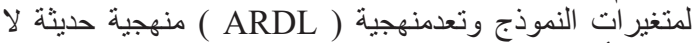

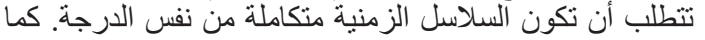

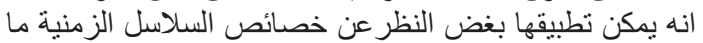

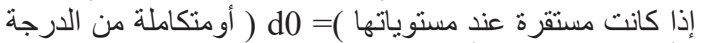

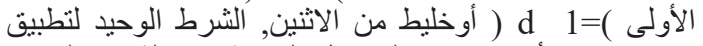

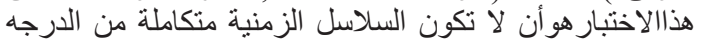

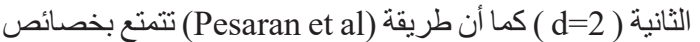

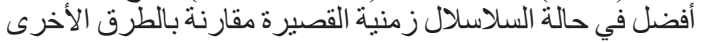

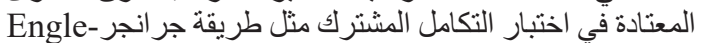

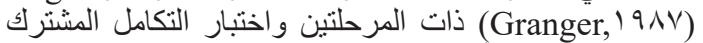

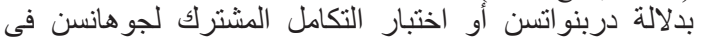
إطار نموذج. (VactorAutoregression Model)

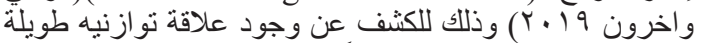

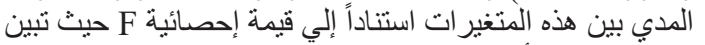

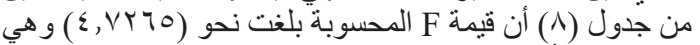

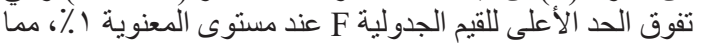

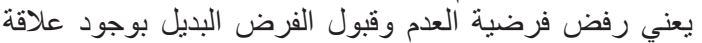

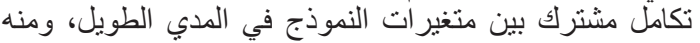

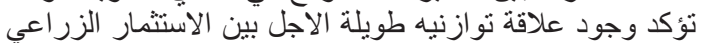

و أهم محدداته كما في معادلة النموذج ريلة رقم (1) (1).
سادساً: محددات الاستثمار الزراعي المصري باستخدام اختبار التكامل المشترك و وفقاً لمنهجية نموذ الزي الانحدار الذاتي للإبطاء

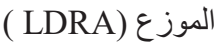

يختص هذا الجزء من البحث التعرف علي أهم العوامل

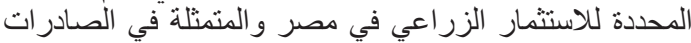

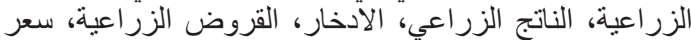

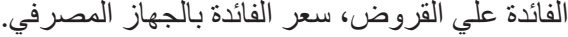
يمكن صياًغة نموذج الاستثمار الزرة اعي على النى النحو التالي : $\mathrm{Y}=\mathrm{F}\left(\mathrm{X}_{1}, \mathrm{X}_{2}, \mathrm{X}_{3}, \mathrm{X}_{4}, \mathrm{X}_{5}, \mathrm{X}_{6}\right)(1)$

تشيث: تشير إلي الصـادر ات الزئي الزراعية.

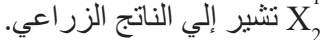
تشير إلي قيمة الادخار.

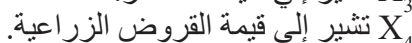

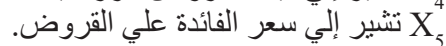

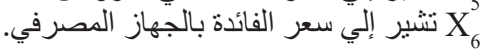

لتقدير هذه النموذج يلزم إجراء بعض الاختبار ات الخاصة

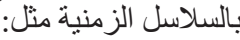

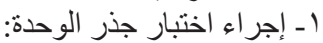

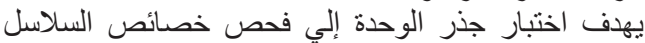

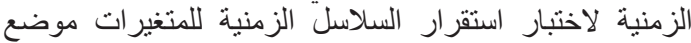

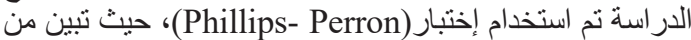

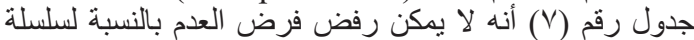

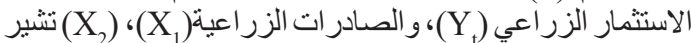

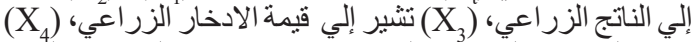

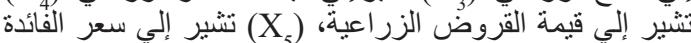

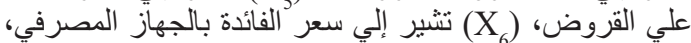

جدول V. يوضح نتائج اختبار (Phillips-Perron) لأهم العوامل المؤثرة علي الاستثمار الزراعي المصري

\begin{tabular}{|c|c|c|c|c|c|c|}
\hline \multicolumn{3}{|c|}{$1^{\text {st }}$} & \multicolumn{3}{|c|}{ Level } & \multirow[b]{2}{*}{ المتغير } \\
\hline None & $\begin{array}{l}\text { Trend and } \\
\text { Intercept }\end{array}$ & Intercept & None & $\begin{array}{l}\text { Trend and } \\
\text { Intercept }\end{array}$ & Intercept & \\
\hline$-1.61215^{*}$ & $-4.70602^{* *}$ & $-2.1098^{\mathrm{ns}}$ & $2.49933^{\mathrm{ns}}$ & $2.63857^{\mathrm{ns}}$ & $2.9167^{\mathrm{ns}}$ & $Y_{t}$ \\
\hline$-2.7559^{* *}$ & $-5.992^{* *}$ & $-4.0057^{* *}$ & $6.44329^{\mathrm{ns}}$ & $-1.9672^{\mathrm{ns}}$ & $2.4997^{\mathrm{ns}}$ & $\mathrm{x}_{1}$ \\
\hline$-1.5505^{*}$ & $-3.6906^{*}$ & $-2.31768^{\text {ns }}$ & $5.2742^{\mathrm{ns}}$ & $-0.6737^{\text {ns }}$ & $3.6538^{\text {ns }}$ & $\mathrm{X}_{2}$ \\
\hline$-2.5573^{*}$ & $-3.3912^{* *}$ & $-2.4590^{\mathrm{ns}}$ & $1.41422^{\mathrm{ns}}$ & $-0.32411^{\mathrm{ns}}$ & $-1.5694^{\mathrm{ns}}$ & $X_{3}$ \\
\hline$-2.0197^{*}$ & $-2.0471^{*}$ & $-1.95923^{\mathrm{ns}}$ & $-1.11912^{\mathrm{ns}}$ & $0.91908^{\mathrm{ns}}$ & $-1.3793^{\mathrm{ns}}$ & $\mathrm{X}_{4}$ \\
\hline$-3.0838^{*}$ & $-3.91217^{*}$ & $-3.12218^{*}$ & $0.90841^{\mathrm{ns}}$ & $0.53192^{\mathrm{ns}}$ & $0.0652^{\mathrm{ns}}$ & $X_{5}$ \\
\hline$-15.600^{* *}$ & $-14.0467^{* *}$ & $-14.7974^{* *}$ & $-2.75345^{\text {ns }}$ & $-3.88197^{\text {ns }}$ & $-3.9798^{\mathrm{ns}}$ & $X_{6}$ \\
\hline
\end{tabular}


جدول ^. اختبار Bound-Test لوجود علاقة طويلة الأجل بين محددات الاستثمار الزراعي

\begin{tabular}{|c|c|c|}
\hline F-Statistics & $\varepsilon, V Y>0$ & \\
\hline \multicolumn{3}{|c|}{ الحدود الحرجة } \\
\hline Significance & الحد الأدنى I(0) & الحد الأعلى I(1) \\
\hline$\% 1$. & r.t & $r, .9$ \\
\hline$\%$ & $r, 07$ & $r, \leq q$ \\
\hline$\% r, 0$ & $r, \Lambda \Lambda$ & $\Gamma, \wedge \mathrm{V}$ \\
\hline$\% 1$ & $r, r q$ & $\varepsilon, r v$ \\
\hline
\end{tabular}

الترتيب.

نموذج تصحيح الخطأ (ECM) وفقاً لنموذج (ARDL):

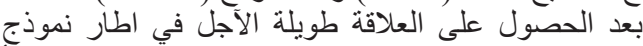

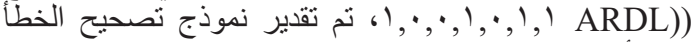
حيث أمكن الحصول علي المعادلة المقدرة التالية.

$\operatorname{DLog} \hat{\mathrm{y}}_{\mathrm{t}}=-1.442 \Delta \log \mathrm{X}_{3 \mathrm{t}}-0.865 \Delta \log \mathrm{X}_{5 \mathrm{t}}+0.0202$ $\Delta \log \mathrm{X}_{6 \mathrm{t}}$

$$
\begin{gathered}
(-6.402)^{* *}(-3.448)^{* *} \quad(2.476)^{\mathrm{ns}} \\
\mathrm{ECM}=-0.93 * *
\end{gathered}
$$

وتشير النتائج للمعادلة رقم (ب) المقدرة في المدي القصير

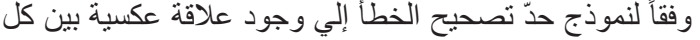

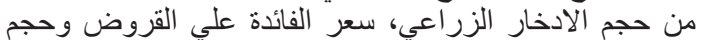

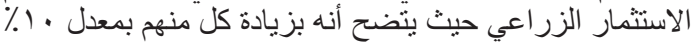

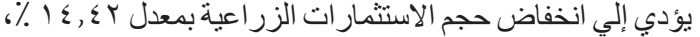

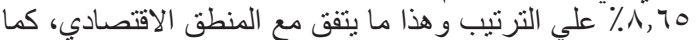

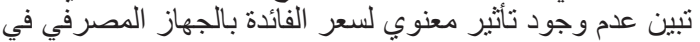

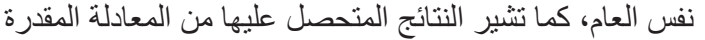

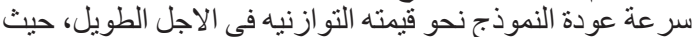

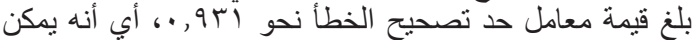

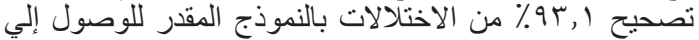
وضع التوازن خلال م ـ ـ ـ سنه.

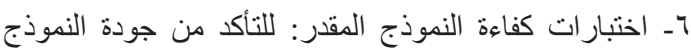

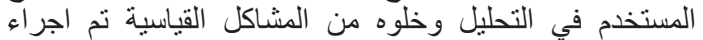
الاختبار ات ألتشخيصية التالية:-

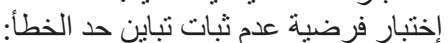

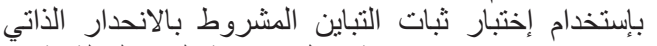

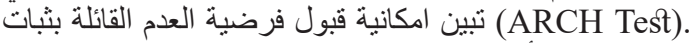

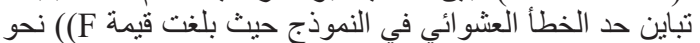

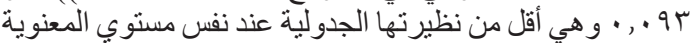

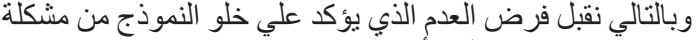

$$
\text { عدم ثبآت تباين حد الخطأ. }
$$

إختبار الارتباط التسلسلي للأخطاء العشوائية (LM Test):

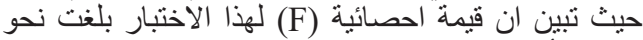

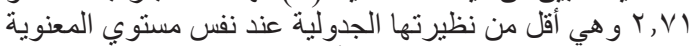

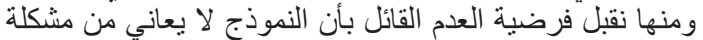
الارنباط الذاتي التسلسلي لبو اقي معادلة الانحدار .

اختبار التوزيع الطبيعي (Normality Test): بالتحقق من التوزيع الطبيعي لبو اقي معادلة الانحدار، تبين من خلال شكل (r) أن قيمة احصائية (Jarque-Bera) بلغت التهن

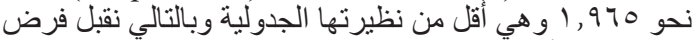

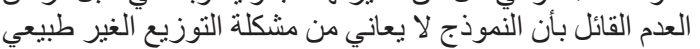

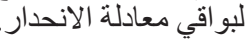

المصدر :نتائج التقدير القياسي باستخدام برنامج E-views.

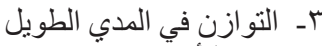

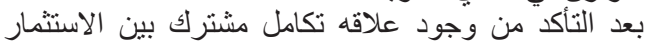

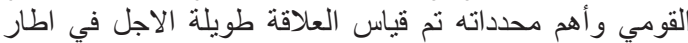

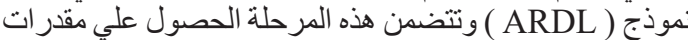
المعلمات في الاجل الطويل، حيث أمكن الحصول لئل علي المعادلة علئ

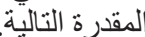

$\log \hat{y}=2.895-0.218 \log \mathrm{X}_{1}+1.552 \log \mathrm{X}_{2}-3.816$ $\log \mathrm{X}_{3}+0.72 \log \mathrm{X}_{4}-1.399 \log \mathrm{X}_{5}+0.131 \log \mathrm{X}_{6}$ (2)

$(3.322) * *(-.215)^{\mathrm{ns}}(6.484) * *(-4.71) * *(2.544) * *(-$ $2.435)^{*}(2.053)^{\mathrm{ns}}$

$\mathrm{R}-2=0.88 \quad \mathrm{~F}=56.93^{*}$ حيث أن : ب أن تثثير إلي لو غاريتم الاستثمار الزر اعي.

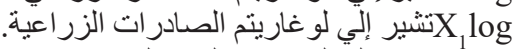

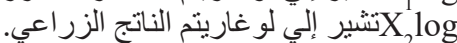
تشير إلي لو غاريتم قيمة الادخار الزراعي. لتئي.

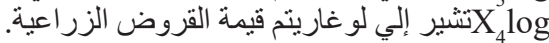

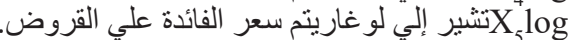

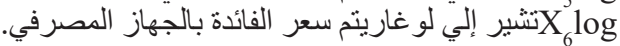

تشير النتائج المتحصل عليها من المعادلة المقدرة رقم (r) لقياس العلاقة طويلة الاجل بين الاستثمار القومي وأهم أهم العو امل

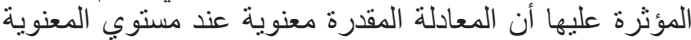

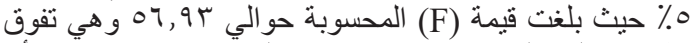

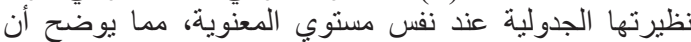

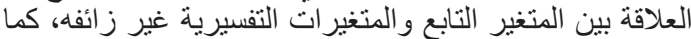

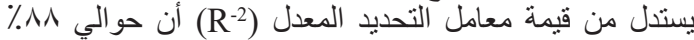

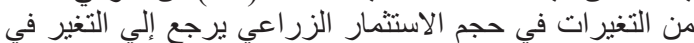
المتغير ات المستقلة مجتمعة التي تضمنها النموذج الني

كما تثير النتائج بالنموذج المقدر إلي عدم ثبوت المعنوية

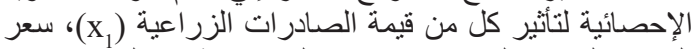

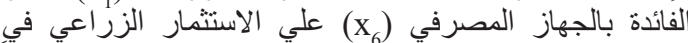

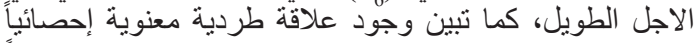

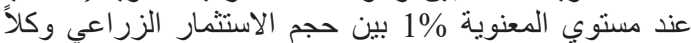

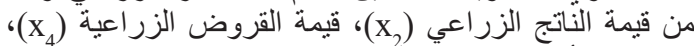

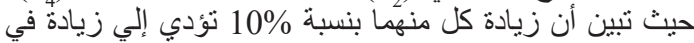

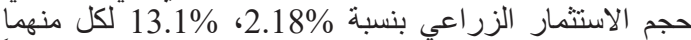

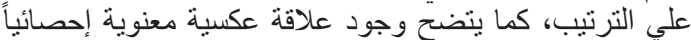

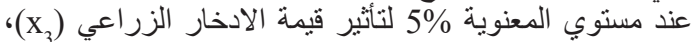

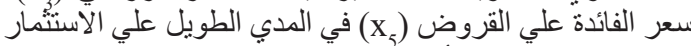

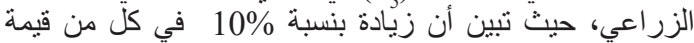

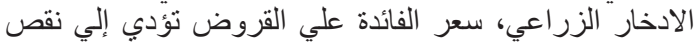

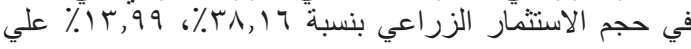




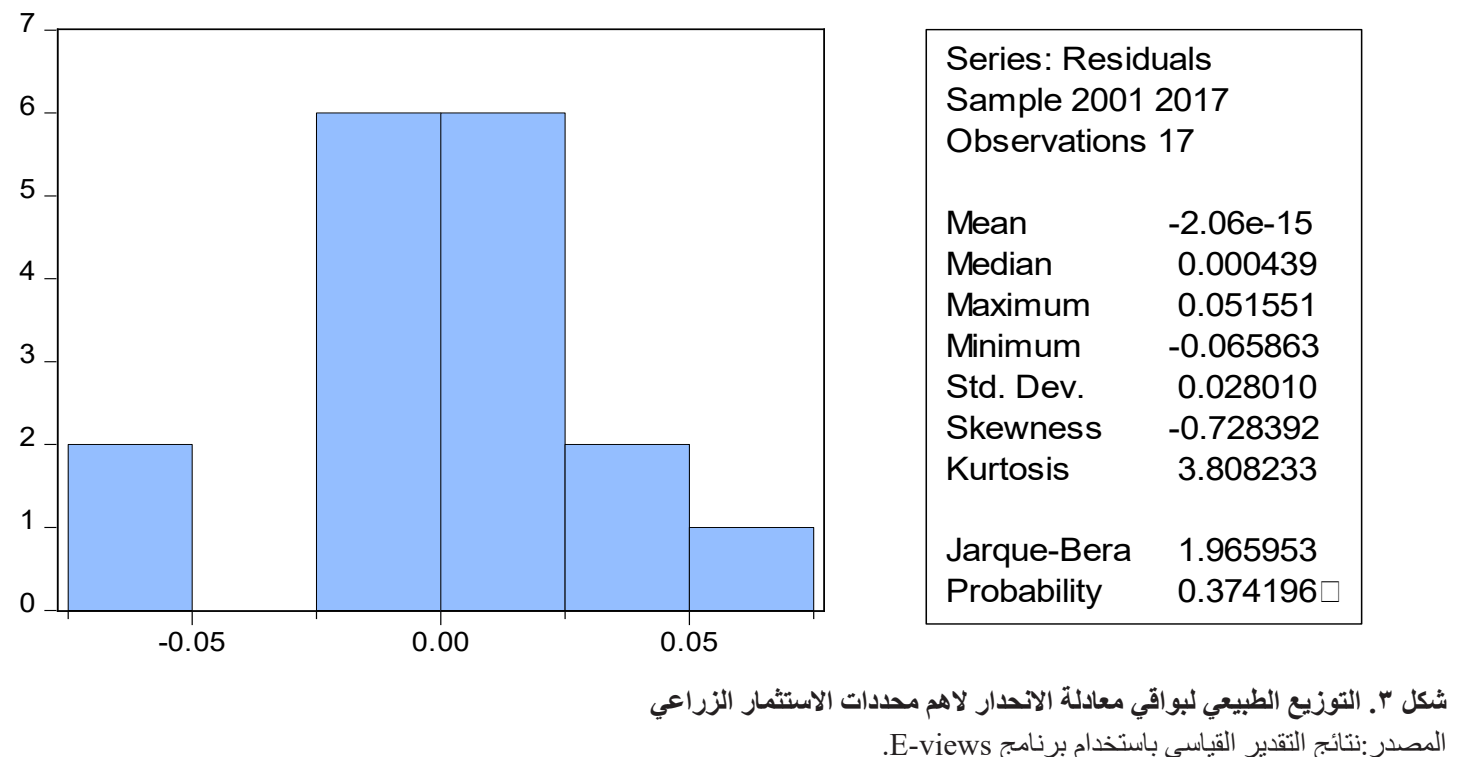

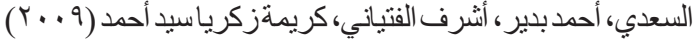

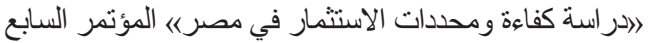

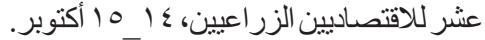

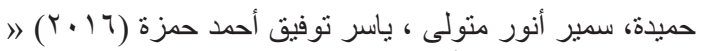

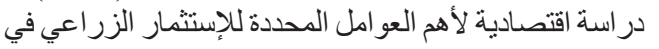

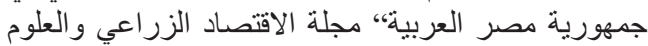

الإجتماعية، جامعة المنصورة، المجلد (V)، العدد (1) (1).

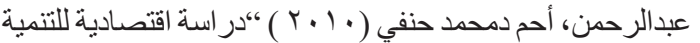

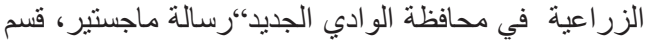

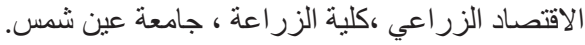

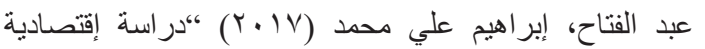

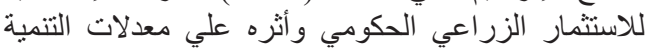

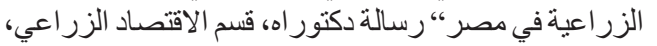

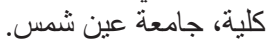

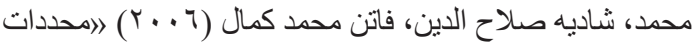

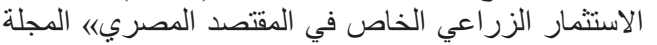

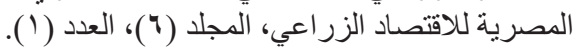

موسي، مراد ذكي، أشرف عبدالله الفتياني، ياسمين عماد الدين

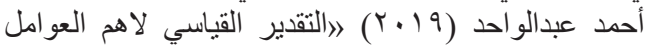

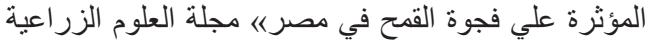

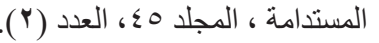
وزارة التخطيط والإصلاح الإداري تقرير متابعة خطة التنمية الإقتصادية و الإجنماعية.
في ضوء ما نوصل إلبه البحث من نتائج فإنه يمكن عرض التوصيات الآتية :

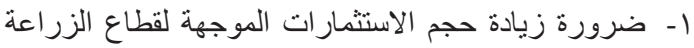

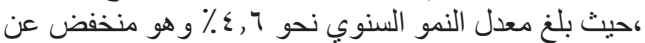

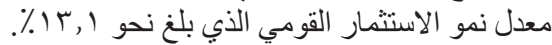

r- ضرورة تدخل الدولة لزيادة حجم الاستثمارات الزراعية

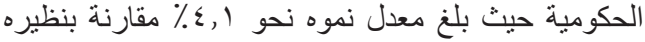

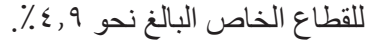

r- ضرورة زيادة القروض الزراعة بلة حيث تبين أن زيادة

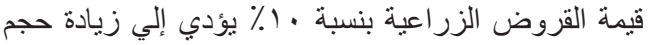

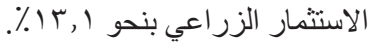

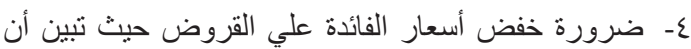

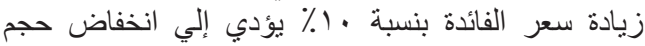

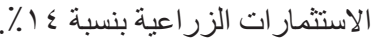




\title{
An Econometric Study of The Determinants of Agricultural Investment in Egypt
}

Fathia Radwan Salem, Mourad Z. Moussa and Amira Eldesoki Taha Ahmed

Department of Agricultural Economics. Faculty of Agriculture Kafr El- Sheikh,Egypt

\begin{abstract}
GRICULTURAL investment is the basis for the success of agricultural development, as it is the main pillar to increase production as well as increase income and create more jobs, Investment is a flowing variable that has an active role in finding solutions to the problems of the Egyptian economy as well as absorbing some of the non-working manpower to contribute to reducing the problem of unemployment. Its importance comes at the forefront of national goals to face the growing economic problems.It also notes the decline of agricultural investments, so the research aims to identify the determinants of agricultural investment using the method of joint integration, The research relied on many research methods, including the descriptive and quantitative methods of the variables under study. The research relied on the published and unpublished secondary data. The results indicate that agricultural investment represents about 24.7 billion pounds, which represents $6 \%$ of the total investment. Agricultural GDP, the value of savings, the value of agricultural loans, the research recommends The need to increase the volume of investments directed to the agricultural sector, where the annual growth rate of about $4.6 \%$, which is lower than the rate of growth of national investment, which reached about $13.1 \%$, And the need to increase agricultural loans where it was found that increasing the value of agricultural loans by $10 \%$ leads to increase the volume of agricultural investment by about $13.1 \%$.
\end{abstract}

Key words:ARDL, Agricultural investment, GDP, Agricultural output, Agricultural exports, Agricultural loans.

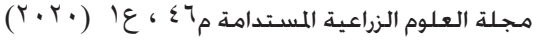

